\title{
Thrombospondin-I Serum Levels In Hypertensive Patients With Endothelial Dysfunction After One Year Of Treatment With Perindopril
}

This article was published in the following Dove Press journal:

Drug Design, Development and Therapy

\begin{abstract}
Valentina Buda, ${ }^{1, *}$
Minodora Andor, (iD) $2, *$

Carmen Cristescu, ${ }^{1} *$

Mirela Cleopatra Tomescu, (iD) ${ }^{2}$

Danina M Muntean, ${ }^{3}$

Dana Emilia Bâibâță, 4,5

Diana Aurora Bordejevic, (iD) 4,5

Corina Danciu, ${ }^{6}$ Olivia Dalleur, ${ }^{7}$

Dorina Coricovac, ${ }^{8}$

Zorin Crainiceanu, ${ }^{9}$ Anca Tudor, ${ }^{10}$

lonut Ledeti, " Lucian Petrescu ${ }^{4,5}$

'Department of Pharmacology and Clinical Pharmacy, Faculty of Pharmacy, "Victor Babeș"

University of Medicine and Pharmacy, Timisoara

30004 I, Romania; ${ }^{2}$ Department of Medical

Semiotics, Faculty of Medicine, "Victor Babess"

University of Medicine and Pharmacy, Timisoara

30004I, Romania; ${ }^{3}$ Department of

Pathophysiology, Faculty of Medicine, "Victor

Babes" University of Medicine and Pharmacy,

Timisoara 30004I, Romania; ${ }^{4}$ Department of

Cardiology VI, Faculty of Medicine, "Victor Babeș"

University of Medicine and Pharmacy, Timisoara

30004I, Romania; ${ }^{5}$ Cardiovascular Diseases

Institute, Timisoara 300310, Romania;

'Department of Pharmacognosy, "Victor Babeș”

University of Medicine and Pharmacy, Timisoara

30004I, Romania; ${ }^{7}$ Clinical Pharmacy Research

Group, Louvain Drug Research Institute,

Université Catholique De Louvain, Woluwe-Saint-

Lambert 1200, Bruxelles, Belgium; ${ }^{8}$ Department of

Toxicology, Faculty of Pharmacy, "Victor Babeș"

University of Medicine and Pharmacy, Timisoara

30004I, Romania; ${ }^{9}$ Department of Plastic and

Reconstructive Surgery, Faculty of Medicine,

"Victor Babeș" University of Medicine and

Pharmacy, Timisoara 30004I, Romania;

${ }^{10}$ Department of Statistics and Biomedical

Informatics, Faculty of Medicine, "Victor Babeș"

University of Medicine and Pharmacy, Timisoara

30004I, Romania; "Department of Physical

Chemistry, Faculty of Pharmacy, "Victor Babeș"

University of Medicine and Pharmacy, Timisoara

30004I, Romania
\end{abstract}

*These authors contributed equally to this work

Correspondence: Valentina Buda

Corina Danciu Faculty of Pharmacy, "Victor

Babeș" University of Medicine and Pharmacy, 2

Eftimie Murgu Street, Timisoara 30004I,

Romania

$\mathrm{Tel}+40-256-494-604$

Fax +40-256-494-604

Email buda.valentina.oana@gmail.com
Background: Thrombospondin-1 (TSP-1) is a matricellular functional protein of the extracellular matrix. As it is not constitutively present extracellularly, its secretion is enhanced in several situations, namely injury, chronic pathology, tissue remodeling, angiogenesis, and aging. Over the last decade, TSP-1 has been reported to be involved in complex and opposing biological effects on vasculature in the context of NO signaling. Several studies have reported high patient TSP-1 plasma levels, indicating that the protein can potentially serve as a prognostic marker for pulmonary arterial hypertension.

Materials and methods: Here, we aimed to quantify TSP-1 serum levels in hypertensive patients with endothelial dysfunction before and after one year of treatment with Perindopril (an antihypertensive drug with vasoprotective properties).

Results: After one year of treatment, TSP-1 levels increased in hypertensive patients compared to baseline (T0: $8061.9 \pm 3684.80$ vs $\mathrm{T} 1: 15380 \pm 5887 \mathrm{ng} / \mathrm{mL}, \mathrm{p}<0.001$ ) and compared to non-hypertensive controls $(9221.03 \pm 6510.21 \mathrm{ng} / \mathrm{mL})$. In contrast, pentraxin3 plasma levels were decreased after one year of Perindopril treatment in both hypertensive (T0: $0.91 \pm 0.51$ vs $\mathrm{T} 1: 0.50 \pm 0.24 \mathrm{ng} / \mathrm{mL}, \mathrm{p}<0.001)$ and control group $(1.36 \pm 1.5 \mathrm{ng} / \mathrm{mL})$ patients, although flow-mediated vasodilation and intima-media thickness assessment parameters were not significantly changed. Systolic and diastolic blood pressure values as well as levels of fibrinogen, high-sensitivity C-reactive protein, triglycerides, and alanine aminotransferase were found to be significantly lower after one year of treatment with Perindopril. High levels of TSP-1 strongly correlated with platelet count (positive), lymphocytes (positive), red cell distribution width-CV (positive), systolic blood pressure (negative), and mean corpuscular hemoglobin (negative) after one year of treatment. Blood urea nitrogen was found to be a protective factor for TSP-1, while glucose and heart rate were found to be risk factors prior to and after treatment.

Keywords: TSP-1, essential arterial hypertension, endothelial function, ACE inhibitors

\section{Introduction}

Thrombospondin-1 (TSP-1), the first natural inhibitor of angiogenesis to be identified in 1971, is a large, homotrimeric $450 \mathrm{kDa}$ glycoprotein stored in $\alpha$-granules of platelets and is released upon platelet activation. ${ }^{1,2}$ Several other cellular sources of TSP-1 have since been identified, including endothelial cells (EC), ${ }^{3}$ vascular smooth muscle cells (VSMCs), ${ }^{4}$ epithelial cells, ${ }^{5}$ keratinocytes, ${ }^{6}$ fibroblasts, ${ }^{7}$ innate immune cells (such as $\mathrm{CD} 4{ }^{+} \mathrm{CD} 25^{-}$T-cells), ${ }^{8}$ dendritic cells, ${ }^{9}$ and macrophages. ${ }^{10}$ TSP-1 can also be detected in embryonic heart, lung, and intestinal tissue from the 10th day onward. ${ }^{11}$ In addition, this protein plays significant roles in cellular 
adhesion, migration, and proliferation, as well as angiogenesis, apoptosis, and cytoskeletal organization. ${ }^{12}$ It has been reported to interact with several key proteins involved in the maintenance of vascular structure and primary hemostasis by promoting platelet aggregation. ${ }^{1}$ It has been classified as a counter-adhesive protein due to its capacity to inhibit cell-substrate adhesion.

Multiple domain sequences interact with several cell receptors and trigger opposing effects in various tissues. Among the TSP-1 cellular receptors, the most studied to date have been CD36and CD47 (known also as integrin associated protein [IAP]). ${ }^{1}$

CD36 is the principal receptor of TSP-1 expressed in platelets, monocytes, ECs, VSMCs, adipocytes, and macrophages. The anti-angiogenic and anti-inflammatory properties of TSP-1 are attributed to the interaction between TSP-1 and CD36. ${ }^{1}$

CD47 is another receptor for TSP-1 and is expressed by ECs, platelets, erythrocytes, VSMCs, epithelial cells, T-cells, and polymorphonuclear cells. It binds to the C-terminal domain of TSP-1 inducing several, often opposing effects on cell survival. By binding to TSP-1, CD47 regulates effects exerted by nitric oxide (NO), inhibits the release of interleukin-2 (IL-2), induces leucocyte apoptosis, prevents phagocytic clearance, and inhibits stem cell self-renewal. ${ }^{1,13}$

In ECs, TSP-1 binds to CD36 or CD47 to inhibit guanylyl cyclase (GC) solubility, the subsequent activation of nitric oxide, and the onset of inflammatory reactions (due to the blocking of leucocyte adhesion and activation as well as decreasing angiogenesis). ${ }^{13}$

TSP-1 is also involved in the process of platelet aggregation and adhesion to ECs. ${ }^{1}$ It has the capacity to activate TGF- $\beta 1$ and exerts pleiotropic effects on the cardiovascular system - either protective, via its anti-atherogenic and plaque-stabilizing properties, or pro-inflammatory. ${ }^{1}$

Conflicting evidence regarding the effects of TSP-1 on VSMCs has been reported. Indeed, TSP-1 has been found to either stimulate proliferation in vitro and in vivo ${ }^{14,15}$ or induce anti-proliferative effects by binding to CD47. ${ }^{16}$ Moreover, both exogenous and endogenous TSP-1 inhibits NO-dependent signaling in VSMCs via a cGMP signaling CD36-dependent pathway. ${ }^{4}$ With respect to TSP-1 effects on the ECs, recent findings suggest that TSP-1 is involved in the inhibition of cell-cell and cell-matrix interaction, as well as in enhancing or inhibiting cell permeability, due to the capacity of ECs to respond to, but also to produce, TSP-1. ${ }^{1}$ Vila et al assessed the relationship between endothelial dysfunction, angiogenesis, and circulating inflammatory biomarkers in patients with chronic heart failure, concluding that low levels of TSP-1 are a consequence of the endothelial repair process. ${ }^{17}$

The complex biological effects of TSP-1 on vasculature are thus likely interrelated to the specific pathologic condition investigated. TSP-1 has recently been recently proposed to be a potential prognostic marker for pulmonary arterial hypertension (PAH) by several studies that reported high plasma levels of TSP-1 in PAH patients compared to controls. ${ }^{18-20}$ Here, we aimed to quantify TSP-1 levels in hypertensive patients with endothelial dysfunction before and after one year of treatment with Perindopril, an antihypertensive drug with vasoprotective properties.

\section{Materials And Methods}

A prospective, comparative study was carried out between January 2015 and June 2016 in the Cardiology Clinic of Timisoara City Hospital, Romania.

\section{Patient Selection}

A total of 142 patients were enrolled in this study. A total of 76 patients with normal blood pressure values (control group) and 66 hypertensive patients treated monotherapeutically with different antihypertensive drugs other than the ACEi or ARBs. All patients studied were matched by age and sex.

Patients included in the control group were assessed for essential arterial hypertension; 24-hr monitoring devices did not suggest this diagnosis.

The inclusion criteria for the hypertensive patient group were as follows: age $>18$ years and an established diagnosis of primary arterial hypertension. Patients who presented other confirmed pathologies such as atherosclerotic disease, coronary artery disease, heart failure, diabetes, renal and hepatic pathologies, asthma, or acute and chronic inflammatory diseases were excluded from the study. In addition, patients treated with ACEi or ARB medications were excluded from this study (due to their similar mechanisms in reversing endothelial dysfunction) for the purposes of ensuring greater finding accuracy and precision.

All recruited patients completed informed consent forms and underwent thorough medical screening including collection a family medical history and laboratory/ paraclinical investigations. 
After medical evaluation, prior antihypertensive treatment was changed to Perindopril (administered in either $5 \mathrm{mg}$ or $10 \mathrm{mg}$ doses based on individual patient blood pressure levels) due to either original antihypertensive treatment insufficiently controlling blood pressure, patient complaints of side effects, or the manifestation of other risk factors under previous treatment regimens.

All hypertensive patients were evaluated at baseline $T_{0}$ (when treated with other antihypertensive drugs) and $\mathrm{T}_{1}$ (after one year of treatment with Perindopril).

\section{Laboratory Analysis}

Venous blood samples were collected (catheter size: 22 gauge) in the morning (8:30 am), in a temperature-controlled room $\left(22^{\circ} \mathrm{C}-24^{\circ} \mathrm{C}\right)$ of the hospital, at least $8 \mathrm{hrs}$ after their last consumption of food, sweet fluids, coffee, or tobacco use during a period when no physical effort was made. The standard biochemical tests (eg, complete blood count, total cholesterol, creatinine, and hepatic transaminase levels) were completed in the hospital according to standard procedures. Plasma levels of pentraxin-3 (PTX3), high-sensitivity $\mathrm{C}$ reactive protein (hs-CRP), and serum TSP-1 levels were assessed by the Bioclinica SA Laboratory in Timisoara. Plasma concentrations of PTX3 and serum TSP-1 levels were assessed via ELISA; kits were provided by R\&D Systems. Plasma levels of hs-CRP were assessed via application of the highly sensitive immunoturbidimetric method; kits were provided by Abbot Diagnostic Company. As all protocols followed are standardized, we have taken the liberty of omitting them here.

Storage containers of venous blood samples were manipulated in the laboratory within 30 mins of collection. The normal range of TSP-1 serum levels provided by the laboratory was $8794-28335 \mathrm{ng} / \mathrm{mL}$.

\section{Arterial Pressure}

Blood pressure was measured in the morning after $30 \mathrm{mins}$ of rest, while in a supine position, at the right brachial artery, and in the same temperature-controlled room. The final value of arterial pressure was obtained from the arithmetic mean of 3 consecutive measurements at 5-min intervals.

The presence or absence of endothelial dysfunction was assessed using not only a flow-mediated dilation measurement dependent of endothelial status (FMD) - a functional assessment, but also by assessing intimal-medial thickness (IMT) - a structural assessment.

\section{Flow-Mediated Dilation Assessment}

FMD assessment was performed after patient fasting for a minimum of $8 \mathrm{hrs}$. During that time period, patients were not permitted to consume any type of foods, coffee, tea, or vitamins. In addition, no physical effort, tobacco use, or vasoactive drug use was permitted. After 10 mins of rest in a supine position while in a soundproof room, brachial arterial diameter was measured using high-resolution ultrasonography (General Electric VIVID S5) equipped with a $9 \mathrm{MHz}$ linear transducer. Longitudinal scans of the brachial artery were performed approximately $5 \mathrm{~cm}$ proximal to the antecubital fossa. Vessel diameters were recorded at baseline and after 5 mins of reactive hyperemia induced by the deflation of the blood pressure cuff, previously inflated to $50 \mathrm{mmHg}$ above the patient's systolic blood pressure. FMD values were calculated as follows:

$$
\begin{aligned}
& \% \mathrm{FMD}=\left[\left(\begin{array}{l}
\text { reactive hyperemia diameter } \\
- \text { standard diameter }
\end{array}\right) / \text { standard diameter }\right] \\
& \times 100
\end{aligned}
$$

We considered FMD to be: ${ }^{21-23}$

- "normal" - if the brachial artery was dilated $20 \%$ more than the initial standard diameter

- "impaired" and thus with endothelial dysfunction - if the brachial artery did not dilate $20 \%$ more than the initial standard diameter

Three measurements were performed for each patient and the arithmetic mean was noted.

\section{Intima-Media Thickness Assessment}

Tunica intima and media thickness was assessed for IMT evaluation. This technique is used to assess the presence of atherosclerotic disease and track its progression, regression, and constancy of the atherosclerotic process.

Baseline IMT was assessed bilaterally at the common carotid artery $2 \mathrm{~cm}$ inferior to the internal and external carotid artery bifurcation. A high-resolution ultrasonography system (General Electric VIVID S5) equipped with a $9 \mathrm{MHz}$ linear transducer was used in B mode. Patients were examined in a supine position and the IMT value was given online by software built into the medical system. Normal (less than or equal to $0.9 \mathrm{~mm}$ ) and high (greater than $1.2 \mathrm{~mm}$ ) values were considered to confirm atherosclerosis and associate with a high risk of cardiovascular diseases. 


\section{Echocardiography}

Echocardiography was performed using the GE VIVID S5 medical system to assess effects of hypertension on cardiac structure and function. Patients with ejection fractions (EF) of $<40 \%$ were excluded from the study. Parameters evaluated included the diameter of the left atrium (DLA), dimensions of the posterior interventricular septum (IVS), the left ventricular end-diastolic diameter (EDLV), and the ejection fraction (EF).

All measurements were performed by the same certified cardiologist.

\section{Statistical Data Analyses}

Data are presented as mean \pm standard deviation (SD). Differences between related groups were analyzed using the Wilcoxon Signed Ranks test while differences between independent groups were analyzed using the MannWhitney test. Correlations between TSP-1 and other variables were studied using multivariable regression analysis; correlation strength was obtained by applying Spearman correlation coefficient. Risk analysis for TSP-1 values greater than 8000 was performed by calculating the odds ratio of nominal variables. Statistical tests were performed using SPSS v.17; $<<0.05$ was considered statistically significant.

\section{Compliance With The Ethical Standards}

The present study was approved by the Ethical Committee of the Victor Babes University of Medicine and Pharmacy, Timisoara, Romania, no. 7/2016. All procedures performed in this study with human participants were in accordance with the ethical standards of the institutional research committee and with the 1964 Helsinki Declaration and its later amendments or comparable ethical standards.

\section{Results}

A total of 76 non-hypertensive patients (41 men and 36 women) were enrolled in the control group; mean age was $55.7 \pm 14.40$. A total of 66 hypertensive patients (36 men and 30 women) were enrolled in the study group; mean age was $56.34 \pm 10.50$.

Demographic and laboratory characteristics of patients enrolled in this study are presented in Table 1. Perindopril significantly decreased systolic blood pressure after one year of treatment $(\mathrm{p}<0.001)$. Beneficial effects were also recorded with respect to the severity of low-grade inflammation assessed by fibrinogen ( $p=0.022)$, hs-CRP $(p=0.006)$, and alanine aminotransferase (ALAT) $(\mathrm{p}<0.001)$ levels. Interestingly, a significant increase in creatinine levels and a non-significant elevation of BUN were also observed after one year of treatment.

Table I Blood Pressure And Standard Laboratory Investigation Values At T0 (baseline) And TI (after One Year Of Treatment With Perindopril)

\begin{tabular}{|c|c|c|c|c|c|c|}
\hline Parameters & Control $(n=76)$ & T0 $(n=66)$ & TI $(n=66)$ & $\begin{array}{l}\text { T0 vs TI p } \\
\text { (Wilcoxon } \\
\text { Signed Rank) }\end{array}$ & $\begin{array}{l}\text { Control vs T0 p } \\
\text { (Mann- } \\
\text { Whitney) }\end{array}$ & $\begin{array}{l}\text { Control vs TI p } \\
\text { (Mann- } \\
\text { Whitney) }\end{array}$ \\
\hline $\mathbf{S B P}(\mathrm{mmHg})$ & $123.20 \pm 17.48$ & $143.17 \pm 16.10$ & $|3| .82 \pm 14.40$ & $<0.001$ & $<0.001$ & 0.002 \\
\hline DBP (mmHg) & $76.67 \pm 9.37$ & $87.89 \pm 7.85$ & $84.09 \pm 13.66$ & 0.013 & $<0.001$ & $<0.001$ \\
\hline HTN duration (months) & - & $57.80 \pm 51.74$ & $68.81 \pm 56.12$ & 0.548 & - & - \\
\hline Heart rate (beats/min) & $70.61 \pm 7.64$ & $74.53 \pm 10.75$ & $70.27 \pm 8.59$ & 0.012 & 0.015 & 0.802 \\
\hline ALAT(U/L) & $38.11 \pm 13.88$ & $38.34 \pm 7.84$ & $33.81 \pm 6.11$ & $<0.001$ & 0.896 & 0.017 \\
\hline ASAT(U/L) & $24.46 \pm 8.56$ & $21.98 \pm 5.70$ & $22.36 \pm 0.71$ & 0.685 & 0.042 & 0.096 \\
\hline Cholesterol(mg/dL) & $193.11 \pm 52.02$ & $|98.7| \pm 47.70$ & $189.18 \pm 53.90$ & 0.109 & 0.507 & 0.660 \\
\hline Triglycerides $(\mathrm{mg} / \mathrm{dL})$ & $130.39 \pm 67.73$ & $105.89 \pm 23.27$ & $98.09 \pm 41.65$ & 0.043 & 0.004 & 0.001 \\
\hline Glucose(mg/dL) & $106.36 \pm 41.34$ & $101.71 \pm 9.15$ & $102.91 \pm 11.98$ & 0.843 & 0.343 & 0.489 \\
\hline Creatinine(mg/dL) & $0.84 \pm 0.24$ & $0.89 \pm 0.16$ & $1.04 \pm 0.38$ & 0.006 & 0.918 & $<0.001$ \\
\hline Potassium(mmol/L) & $4.36 \pm 0.40$ & $4.28 \pm 0.45$ & $4.35 \pm 0.44$ & 0.079 & 0.285 & 0.952 \\
\hline ESR $(\mathrm{mm} / \mathrm{hr})$ & $8.72 \pm 5.48$ & $11.89 \pm 4.78$ & $|2.18 \pm 7.4|$ & 0.844 & $<0.001$ & 0.002 \\
\hline Fibrinogen(mg/dL) & $307.57 \pm 58.86$ & $328.03 \pm 62.03$ & $302.35 \pm 59.61$ & 0.022 & 0.046 & 0.601 \\
\hline hs-CRP (mg/dL) & $0.24 \pm 0.35$ & $0.17 \pm 0.12$ & $0.12 \pm 0.11$ & 0.006 & 0.004 & 0.129 \\
\hline BUN (mg/dL) & $19.39 \pm 6.90$ & $16.53 \pm 6.30$ & $18.00 \pm 7.92$ & 0.362 & 0.011 & 0.269 \\
\hline
\end{tabular}

Note: Data are represented as mean $\pm \mathrm{SD}$. Bold font indicates statistical significance.

Abbreviations: SBP, systolic blood pressure; DBP, diastolic blood pressure; ALAT, alanine aminotransferase (GTP); ASAT, aspartate aminotransferase (GOT); ESR, erythrocyte sedimentation rate; Hs-CRP, high-sensitivity C-reactive protein; BUN, blood urea nitrogen. 
Table 2 PTX3, TSP-I, FMD, And IMT Values In Control And Study Groups At To (baseline) And T, (after One Year Of Treatment)

\begin{tabular}{|c|c|c|c|c|c|c|}
\hline Biochemical Markers & $\begin{array}{l}\text { Control } \\
(n=76)\end{array}$ & T0 $(n=66)$ & TI $(n=66)$ & $\begin{array}{l}\text { T0 vs TI p } \\
\text { (Wilcoxon Signed } \\
\text { Rank) }\end{array}$ & $\begin{array}{l}\text { Control vs T0 p } \\
\text { (Mann-Whitney) }\end{array}$ & $\begin{array}{l}\text { Control vs TI p } \\
\text { (Mann-Whitney) }\end{array}$ \\
\hline $\begin{array}{l}\text { Pentraxin-3 (ng/mL) } \\
\text { Thrombospondin- I } \\
(\mathrm{ng} / \mathrm{mL})\end{array}$ & $\begin{array}{l}1.36 \pm 1.5 \\
9221.03 \pm \\
6510.21\end{array}$ & $\begin{array}{l}0.91 \pm 0.51 \\
8061.9 \pm \\
3684.8\end{array}$ & $\begin{array}{l}0.50 \pm 0.24 \\
15380 \pm \\
5887\end{array}$ & $\begin{array}{l}<0.001 \\
<0.001\end{array}$ & $\begin{array}{l}<0.001 \\
0.171\end{array}$ & $\begin{array}{l}0.017 \\
<0.001\end{array}$ \\
\hline $\begin{array}{l}\text { Functional and } \\
\text { structural } \\
\text { parameters }\end{array}$ & $\begin{array}{l}\text { Control } \\
(n=76)\end{array}$ & T0 $(n=66)$ & TI $(n=66)$ & $\begin{array}{l}\text { TO vs TI p } \\
\text { (Wilcoxon Signed } \\
\text { Rank) }\end{array}$ & $\begin{array}{l}\text { Control vs T0 p } \\
\text { (Mann-Whitney) }\end{array}$ & $\begin{array}{l}\text { Control vs TI p } \\
\text { (Mann-Whitney) }\end{array}$ \\
\hline FMD (\%) & $19.11 \pm 8.95$ & $9.91 \pm 8.33$ & $10.38 \pm 7.21$ & 0.822 & $<0.001$ & $<0.001$ \\
\hline CC IMT right (mm) & $0.84 \pm 0.24$ & $0.85 \pm 0.11$ & $0.87 \pm 0.24$ & 0.198 & 0.864 & 0.383 \\
\hline CC IMT left (mm) & $0.85 \pm 0.21$ & $0.91 \pm 0.18$ & $0.87 \pm 0.26$ & 0.164 & 0.065 & 0.558 \\
\hline
\end{tabular}

Note: Data are represented as mean $\pm \mathrm{SD}$. Bold font indicates statistical significance.

Abbreviations: FMD, flow-mediated vasodilatation; CC IMT, common carotid intima-media thickness.

Table 2 summarizes the main biochemical and ultrasound parameters used in the assessment of endothelial dysfunction (ED). Importantly, although ED was present at baseline as assessed by FMD levels $(9.91 \pm 8.33$ vs $19.11 \pm 8.95)$, after one year of Perindopril treatment, the values of investigated parameters remained unchanged, suggesting that no progression of vascular impairment occurred over the course of one year. Nevertheless, statistically significant decreases in PTX3 plasma levels and increases in TSP-1 serum levels, respectively, were recorded after one year of treatment.

After one year of Perindopril treatment, a mild decrease of interventricular septal diameter that did not reach statistical significance was observed. Interestingly, a significant increase in the diameter of the posterior left ventricular wall was noted, with all parameters within normal ranges.

Table 4 presents complete blood cell counts at baseline and after one year of treatment. Although there were some statistically significant alterations after one year of Perindopril treatment such as an increase in the number of leucocytes, decrease in hematocrit and hemoglobin (due to a decrease in lymphocytes and increase in neutrophils), all parameters remained within the normal range and such changes were not considered to be pathological.

Tables 5 and 6 present the analyses performed in order to determine correlations between plasma TSP-1 levels and other parameters both at baseline and after one year of treatment. When patients were treated with other antihypertensive drugs prior to baseline measurements, TSP-1 was found to correlate positively with levels of ALAT and red blood cells ( $r>0.3)$ and negatively with mean corpuscular volume. After one year of Perindopril treatment, TSP-1 correlated positively with several parameters, most strongly positively with platelet count, lymphocytes, and red cell distribution width, and negatively with systolic blood pressure and mean corpuscular hemoglobin.

Table 7 presents the results of logistic regression analyses performed in order to determine correlations between TSP-1 and all studied parameters at baseline. Age $>56.5$ years old was not a risk factor for TSP-1 elevation. There is no significant association between age and TSP-1 $\left(\chi^{2}, \mathrm{p}=0.607\right.$, $\mathrm{OR}=0.79 .95 \%$ interval of confidence $\mathrm{OR}(0.41,1.54))$.

Table 8 presents the results of logistic regression analyses performed in order to determine correlations between TSP-1 and all studied parameters after one year of Perindopril treatment.

Blood urea nitrogen and mean corpuscular hemoglobin were found to be protective factors (both at baseline and after one year of treatment), in contrast with glucose and heart rate, which were found to be risk factors.

As illustrated in Figure 1 and Table 2, upon initiation of Perindopril $\left(\mathrm{T}_{0}\right)$, hypertensive patients had lower TSP-1 serum levels compared with those noted after one year of treatment $\left(\mathrm{T}_{1}\right)$.

As shown in Figure 2, higher TSP-1 serum levels were noted in men both prior to and after one year of treatment $\left(\mathrm{T}_{0}\right.$ and $\left.\mathrm{T}_{1}\right)$.

After one year of Perindopril treatment, patients treated with a $10 \mathrm{mg} /$ day dosage of the drug exhibited the highest TSP-1 serum levels (Figure 3), although the values were very closely related.

\section{Discussion}

As the effects of TSP-1 on endothelial dysfunction have been mostly studied in animal models and less studied in humans 


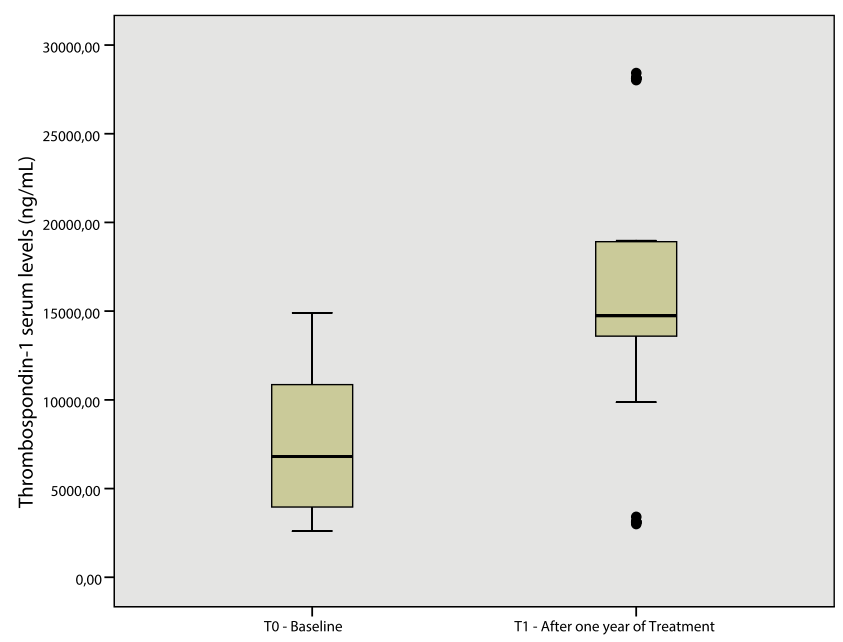

Figure I Thrombospondin-I serum levels at baseline $\left(T_{0}\right)$ and after one year of Perindopril treatment $\left(\mathrm{T}_{1}\right)$.
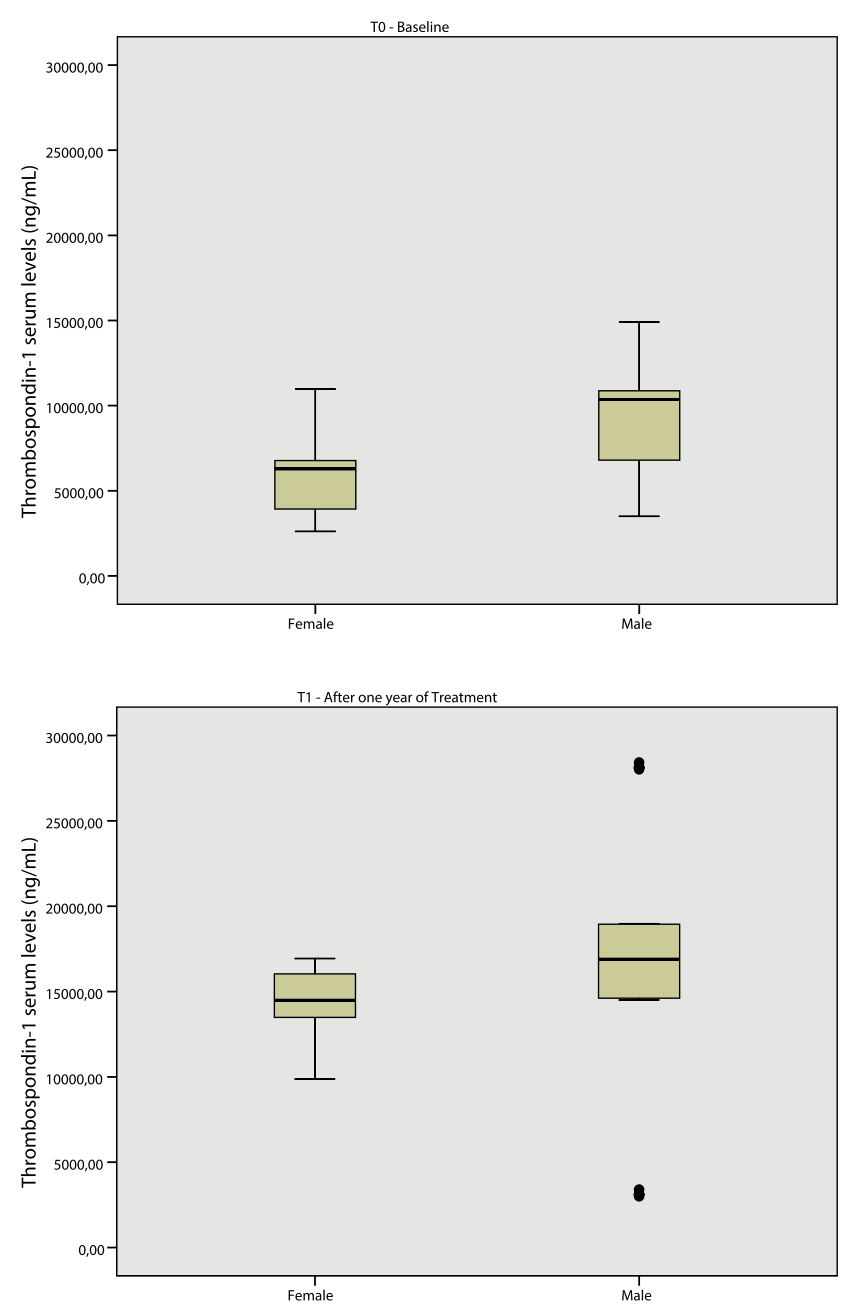

Figure 2 Variation of thrombospondin-I serum levels at baseline $\left(T_{0}\right)$ and after one year of Perindopril treatment $\left(T_{1}\right)$ as a function of patient sex ( $F-$ female; $M-$ male).

needing further research (as the present data are contradictory and incomplete), its role on pulmonary arterial hypertension

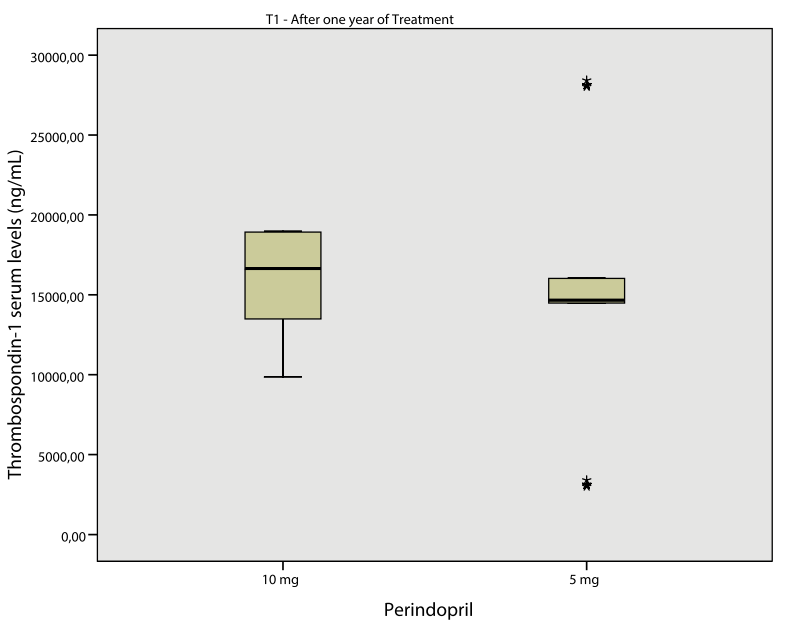

Figure 3 Thrombospondin-I serum levels at $5 \mathrm{mg}$ or $10 \mathrm{mg} /$ day dosages of Perindopril after one year of treatment.

has been more clearly documented over the last 10 years, not only on animal studies but also in human studies. ${ }^{18-20}$

This study is a continuation of our prior work assessing patients under long-term treatment with Perindopril versus other regimens of antihypertensive treatment. ${ }^{24}$ Patients treated with this drug were found to have higher TSP-1 levels compared to patients administered other antihypertensive regimens among hypertensive individuals with endothelial dysfunction.

Perindopril is one of the most frequently prescribed and well-documented ACEi medications. Its use is indicated not only due to its anti-hypertensive properties but also because it exerts cardioprotective effects. Perindopril effectively controls blood pressure via the inhibition of ACE. Perindopril also inhibits the degradation of bradykinin (ie, kinase II) and thus reverses endothelial dysfunction. Via stimulation of B2 receptors, accumulated bradykinin stimulates endogenous kinins to synthesis NO, prostacyclins, and endotheliumderived hyperpolarized factor (EDHF). ${ }^{24}$

TSP-1 has been described as a NO-limiter due to its interaction with CD47, even at very low plasma concentrations. This phenomenon was explained by the fact that it limits the activity of endothelial NO synthase as well as NO signaling via direct sGC targeting and inhibits targets of cGMP. ${ }^{25}$ Moreover, TSP-1 signaling via CD47 stimulates production of reactive oxygen species in hypoxic endothelial cells, vascular smooth muscle cells, and renal tubule epithelial cells. All these effects are likely a consequence of the inhibitory action of TSP-1 on NO and likely depend on both TSP-1 plasma concentrations and the specific pathology involved. As many authors have suggested, further research is required. ${ }^{26-28}$ 
In our present study, Perindopril managed to control (decrease) blood pressure values after one year of treatment, as shown in Table 1, and this was among the reasons why the previous antihypertensive treatment was discontinued. Moreover, heart rate markedly decreased after one year of treatment along with other biochemical parameters such as transaminase, cholesterol, and triglyceride levels (findings were statistically significant); these effects were likely consequences of non-pharmacological treatment implemented together with Perindopril therapy. Potassium levels increased after one year of treatment, as expected, due to the inhibition of aldosterone secretion. Although a modest increase in creatinine was noted, we did not consider this finding abnormal as values remained within normal parameters. Increased creatinine levels were likely due to changes in renal function that occur after inhibition of the rennin-angiotensin-aldosterone system.

After one year of treatment with Perindopril, systemic (reflected by hs-CRP and fibrinogen plasma levels) and local (ie, endothelial) inflammation (reflected by pentraxin-3 plasma levels) significantly decreased.

The TSP-1 serum levels had increased (statistically significant) after one year of treatment with Perindopril (drug with anti-platelet properties ${ }^{29}$ ) the patients' serum levels of TSP-1 being under the normal values that were given by the laboratory: $8794-28335 \mathrm{ng} / \mathrm{mL}$. As already documented, TSP-1 levels measured from serum reflect the TSP-1 release by platelets, compared with TSP-1 plasma levels which reflect the circulating concentrations (secreted biologically active pool). ${ }^{30}$

Pentraxin-3 (PTX-3) is a multimeric inflammatory protein belonging to the same family as $\mathrm{C}$-reactive protein (CRP) and serum amyloid $\mathrm{P}$ component (SAP). It is a marker of vascular damage as its local synthesis is stimulated by TNF, IL-1 $\beta$ (interleukin-1 $\beta$ ), and bacterial products. It is synthesized in endothelial cells, macrophages, myeloid cells, and dendritic cells. This is in contrast to CRP and SAP, which are synthesized in the liver, after the stimulation of IL-6. ${ }^{31,32}$

Our recent study found that hypertensive patients undergoing long-term treatment with Perindopril had decreased plasma levels of soluble endoglin (a glycoprotein involved in cardiovascular homeostasis and a marker of endothelial lesion) compared with patients treated with other classes of drugs, such as $\beta$-blockers, calcium channel blockers, and diuretics. ${ }^{33}$ Here, we found a negative, weak yet statistically significant correlation among TSP-1 and soluble endoglin levels $\left(\mathrm{r}=-0.22, \mathrm{R}^{2}=4.84 \%, \mathrm{p}=0.008\right)$ at baseline compared with a non-significant correlation with PTX-3 levels $(\mathrm{r}=-0.057$, $\mathrm{R}^{2}=0.32 \%, \mathrm{p}=0.502$ ). After one year of Perindopril therapy, we noted a negative, moderate, and statistically significant correlation between TSP-1 and soluble endoglin levels $\left(r=-0.425, R^{2}=18.06 \%, p<0.001\right)$ and a negative, very weak yet statistically significant correlation among TSP-1 and PTX3 levels $\left(\mathrm{r}=-0.153, \mathrm{R}^{2}=2.34 \%, \mathrm{p}=0.048\right)$

With respect to endothelial dysfunction present in all studied patients, pathology neither advanced nor regressed after one year of Perindopril therapy, a finding we consider positive as it appeared to stabilize hypertension. In addition, IMT levels remained very similar after one year of treatment.

Likewise, TSP-1 has been reported to decrease inflammation by binding to the CD47 receptor, a mechanism supported by our study as well, considering that local endothelial inflammation decreased (reflected by plasma PTX-3 levels, which decreased after one year of Perindopril therapy). ${ }^{34}$ The anti-inflammatory actions of TSP-1 were recently confirmed by Meng et al, reporting low serum TSP-1 levels in patients with systemic lupus erythematosus when compared with controls. ${ }^{35}$

As we previously suggested and based on the literature data, ${ }^{4,14-16}$ high plasma TSP-1 levels also exhibit an antiproliferative effect on VSMCs and thus reduce their density via binding of its C-terminal domain to CD47. More precisely, low plasma TSP-1 levels might induce a proliferative effect at the vascular level, while high plasma TSP-1 levels induce an anti-proliferative effect, although the effects may differ depending on the particular pathology concerned. ${ }^{1}$

Bauer et al suggested that TSP-1 plays an important role in maintaining vascular systemic tone; after one year of treatment with Perindopril and based on high levels of TSP-1 along with normalization of blood pressure values, it is likely that vascular systemic tone significantly improved. ${ }^{36}$

Echocardiographic alterations of IVS and PWLV (Table 3) after one year of treatment suggest an active process of cardiac remodeling that involves a decrease in fibrosis. Indeed, an anti-fibrotic effect exerted by TSP-1 was previously reported by other studies. ${ }^{36}$

In summary, our study noted elevated serum TSP-1 levels in hypertensive patients with endothelial dysfunction after one year of treatment with Perindopril, which was likely responsible for a variety of local pleiotropic (ie, anti-inflammatory, anti-proliferative, and anti-fibrotic) effects, as well as the maintenance of systemic vascular tone.

Multivariate regression analysis revealed blood urea nitrogen to be a protective factor (at both $\mathrm{T} 0$ and $\mathrm{T} 1$ ) for TSP-1, although its plasma levels were not significantly 
Table 3 Echocardiographic Parameters At T0 (baseline) And TI (after One Year)

\begin{tabular}{|l|l|l|l|l|l|l|}
\hline $\begin{array}{l}\text { Echocardiographic } \\
\text { Parameters }\end{array}$ & $\begin{array}{l}\text { Control } \\
(\mathbf{n = 7 6 )}\end{array}$ & T0 (n=66) & TI (n=66) & $\begin{array}{l}\text { T0 vs TI p } \\
\text { (Wilcoxon } \\
\text { Signed Rank) }\end{array}$ & $\begin{array}{l}\text { Control vs T0 p } \\
\text { (Mann-Whitney) }\end{array}$ & $\begin{array}{l}\text { Control vs TI p } \\
\text { (Mann-Whitney) }\end{array}$ \\
\hline DLA (mm) & $35.16 \pm 7.29$ & $33.27 \pm 2.19$ & $34.18 \pm 4.39$ & 0.181 & $\mathbf{0 . 0 3 5}$ & 0.329 \\
IVS (mm) & $10.89 \pm 2.81$ & $11.00 \pm 1.71$ & $10.72 \pm 1.22$ & 0.398 & 0.777 & 0.655 \\
PWLV (mm) & $10.05 \pm 1.70$ & $9.52 \pm 1.22$ & $10.18 \pm 1.34$ & $\mathbf{0 . 0 3 8}$ & $\mathbf{0 . 0 4 0}$ & 0.613 \\
EDLV (mm) & $50.42 \pm 7.09$ & $50.00 \pm 3.69$ & $51.18 \pm 5.27$ & 0.313 & 0.652 & 0.466 \\
E.F. (\%) & $56.11 \pm 8.45$ & $55.63 \pm 7.98$ & $55.63 \pm 6.59$ & 0.751 & 0.736 & 0.716 \\
\hline
\end{tabular}

Notes: Data are represented as mean \pm SD. Bold font indicates statistical significance.

Abbreviations: DLA, diameter of left atrium; IVS, diameter of interventricular septum; PWLV, posterior wall of the left ventricle; EDLV, left ventricular end-diastolic diameter; EF, ejection fraction.

Table 4 Complete Blood Count Data

\begin{tabular}{|c|c|c|c|c|c|c|}
\hline Parameters & Control $(n=76)$ & T0 $(n=66)$ & TI $(n=66)$ & $\begin{array}{l}\text { T0 vs TI p } \\
\text { (Wilcoxon } \\
\text { Signed Rank) }\end{array}$ & $\begin{array}{l}\text { Control vs T0 p } \\
\text { (Mann-Whitney) }\end{array}$ & $\begin{array}{l}\text { Control vs TI p } \\
\text { (Mann-Whitney) }\end{array}$ \\
\hline WBC $(\mu \mathrm{L})$ & $7.41 \pm 5.08$ & $5.96 \pm 1.05$ & $6.51 \pm 1.04$ & 0.016 & 0.018 & 0.140 \\
\hline $\mathbf{R B C}(\mu \mathrm{L})$ & $4.69 \pm 0.57$ & $4.82 \pm 0.42$ & $4.4 I \pm 0.5 I$ & $<0.001$ & 0.122 & 0.003 \\
\hline HGB $(g / d L)$ & $14.08 \pm 1.54$ & $14.22 \pm 0.89$ & $13.20 \pm 1.19$ & $<0.001$ & 0.470 & $<0.001$ \\
\hline HCT (\%) & $41.37 \pm 4.09$ & $42.15 \pm 2.40$ & $38.94 \pm 3.93$ & $<0.001$ & 0.160 & $<0.001$ \\
\hline MCV (fL) & $88.51 \pm 4.69$ & $87.52 \pm 3.89$ & $88.41 \pm 4.78$ & 0.232 & 0.181 & 0.909 \\
\hline MCH (pg) & $30.09 \pm 1.49$ & $29.66 \pm 1.07$ & $29.99 \pm 1.47$ & 0.580 & 0.055 & 0.689 \\
\hline MCHC (g/dL) & $34.00 \pm 0.94$ & $33.78 \pm 0.70$ & $33.97 \pm 1.07$ & 0.170 & 0.118 & 0.859 \\
\hline PLT $(\mu \mathrm{L})$ & $244.39 \pm 62.01$ & $261.09 \pm 71.35$ & $256.36 \pm 60.94$ & 0.222 & 0.138 & 0.250 \\
\hline LYMPH(\%) & $30.69 \pm 8.76$ & $35.82 \pm 7.08$ & $27.79 \pm 12.53$ & 0.003 & $<0.001$ & 0.119 \\
\hline MONO(\%) & $8.55 \pm 2.39$ & $10.02 \pm 2.80$ & $8.91 \pm 2.41$ & 0.003 & 0.001 & 0.360 \\
\hline NEUTRO(\%) & $57.82 \pm 10.04$ & $47.93 \pm 14.65$ & $58.03 \pm 8.22$ & $<0.001$ & $<0.001$ & 0.887 \\
\hline EO(\%) & $2.83 \pm 2.92$ & $2.98 \pm 2.14$ & $1.87 \pm 1.32$ & $<0.001$ & 0.733 & 0.011 \\
\hline BASO(\%) & $0.40 \pm 0.18$ & $0.50 \pm 0.28$ & $0.30 \pm 0.22$ & 0.001 & 0.019 & 0.007 \\
\hline LYMPH $(\mu \mathrm{L})$ & $1.96 \pm 0.65$ & $2.11 \pm 0.46$ & $1.98 \pm 0.65$ & 0.279 & 0.115 & 0.865 \\
\hline MONO $(\mu \mathrm{L})$ & $0.56 \pm 0.14$ & $0.59 \pm 0.21$ & $0.58 \pm 0.20$ & 0.506 & 0.226 & 0.366 \\
\hline NEUTRO $(\mu \mathrm{L})$ & $3.84 \pm 1.72$ & $3.05 \pm 0.77$ & $3.78 \pm 0.82$ & $<0.001$ & $<0.001$ & 0.809 \\
\hline EO $(\mu \mathrm{L})$ & $0.17 \pm 0.18$ & $0.17 \pm 0.11$ & $0.12 \pm 0.07$ & 0.010 & 0.984 & 0.038 \\
\hline BASO $(\mu \mathrm{L})$ & $0.03 \pm 0.01$ & $0.03 \pm 0.02$ & $0.02 \pm 0.01$ & 0.008 & 0.092 & 0.033 \\
\hline RDW-CV (\%) & $13.46 \pm 1.08$ & $13.55 \pm 0.70$ & $13.1 \pm 0.99$ & 0.001 & 0.534 & 0.036 \\
\hline RDW-SD (fL) & $41.32 \pm 6.30$ & $42.42 \pm 2.52$ & $41.58 \pm 3.89$ & 0.014 & 0.162 & 0.770 \\
\hline PDW (fL) & $14.63 \pm 2.98$ & $13.43 \pm 1.70$ & $18.23 \pm 12.25$ & $<0.001$ & 0.004 & 0.022 \\
\hline MPV (fL) & $11.41 \pm 1.12$ & $10.99 \pm 0.86$ & $11.10 \pm 1.36$ & 0.030 & 0.017 & 0.157 \\
\hline P-LCR (\%) & $36.36 \pm 8.16$ & $33.20 \pm 6.72$ & $36.18 \pm 6.68$ & $<0.001$ & 0.014 & 0.886 \\
\hline PCT (\%) & $0.27 \pm 0.06$ & $0.28 \pm 0.07$ & $0.28 \pm 0.07$ & 0.690 & 0.213 & 0.290 \\
\hline
\end{tabular}

Notes: Data are represented as mean \pm SD. Statistical significance was considered when $\mathrm{p}<0.05$. Bold font indicates statistical significance.

Abbreviations: WBC, white blood cells; RBC, red blood cells; HGB, hemoglobin; HCT, hematocrit; MCV, mean corpuscular volume; MCH, mean corpuscular hemoglobin; MCHC, mean corpuscular hemoglobin concentration; PLT, platelet count; LYMPH, lymphocytes; MONO, monocytes; NEUTRO, neutrophils; EO, eosinophils; BASO basophils; RDW, red cell distribution width; PDW, platelet distribution width; MPV, mean platelet volume; P-LCR, platelet larger cell ratio; PCT, procalcitonin.

altered after one year, in contrast with TSP-1 (likely due to a short period of observation). On the other hand, glucose and heart rate were found to be risk factors for TSP-1 (at both T0 and T1); a number of other studies previously reported an association between TSP-1 and glucose. ${ }^{37,38}$
Interestingly, the left ventricular end-diastolic diameter and the left atrial diameter were also determined to be risk factors for TSP-1 at baseline (T0), likely due to a certain degree of endothelial dysfunction already present. 
Table 5 Correlations Of TSP-I Plasma Levels With Baseline Data (T0)

\begin{tabular}{|l|l|l|}
\hline Parameters & T0 TSP-I & Correlation \\
\hline ALAT & $\rho=0.416, \mathbf{P}<0.001$ & Positive \\
RBC & $\rho=0.345, \mathbf{P}<0.001$ & Positive \\
MCV & $\rho=-0.348, \mathbf{P}<0.001$ & Negative \\
\hline
\end{tabular}

Abbreviations: ALAT, alanine aminotransferase (GTP); RBC, red blood cells; MCV, mean corpuscular volume.

Table 6 Correlations Of TSP-I Plasma Levels With All Data After One Year Of Treatment (TI)

\begin{tabular}{|c|c|c|}
\hline Parameters & TI TSP-I & Correlation \\
\hline Creatinine & $\rho=-0.385, \mathrm{p}=0.00 \mathrm{I}$ & Negative \\
\hline Potassium & $\rho=-0.479, \mathrm{P}<0.00 \mathrm{I}$ & Negative \\
\hline SBP & $\rho=-0.717, \mathrm{p}<0.00 \mathrm{I}$ & Negative \\
\hline DBP & $\rho=-0.410, \mathrm{p}=0.00 \mathrm{I}$ & Negative \\
\hline RBC & $\rho=0.438, p<0.001$ & Positive \\
\hline $\mathrm{MCH}$ & $\rho=-0.633, \mathrm{p}<0.001$ & Negative \\
\hline $\mathrm{MCHC}$ & $\rho=-0.379, p=0.002$ & Negative \\
\hline PLT & $\rho=0.588, \mathrm{p}<0.00 \mathrm{I}$ & Positive \\
\hline LYMPH\% & $\rho=0.531, p<0.001$ & Positive \\
\hline MONO\% & $\rho=0.362, \mathrm{p}=0.003$ & Positive \\
\hline BASO\% & $\rho=0.394, \mathrm{P}=0.00 \mathrm{I}$ & Positive \\
\hline MONO & $\rho=0.443, p<0.001$ & Positive \\
\hline BASO & $\rho=0.459, \mathrm{p}<0.00 \mathrm{I}$ & Positive \\
\hline RDW-CV & $\rho=0.654, \mathrm{p}<0.001$ & Positive \\
\hline RDW-SD & $\rho=0.362, \mathrm{p}=0.003$ & Positive \\
\hline MPV & $\rho=-0.408, \mathrm{p}=0.00 \mathrm{I}$ & Negative \\
\hline P-LCR & $\rho=-0.460, \mathrm{p}<0.00 \mathrm{I}$ & Negative \\
\hline PCT & $\rho=0.348, \mathrm{p}<0.004$ & Positive \\
\hline
\end{tabular}

Note: Bold font indicates statistical significance.

Abbreviations: SBP, systolic blood pressure; DBP, diastolic blood pressure; RBC, red blood cells; $\mathrm{MCH}$, mean corpuscular hemoglobin; $\mathrm{MCHC}$, mean corpuscular hemoglobin concentration; PLT, platelet count; LYMPH, lymphocytes; MONO, monocytes; BASO, basophils; RDW, red cell distribution width; MPV, mean platelet volume; P-LCR, platelet larger cell ratio; PCT, procalcitonin.
While endogenous TSP-1 is known to function as a pressor, it remains unclear if its pressor effects are due to direct actions or are secondary to other risk factors (angiotensin II stimulates TSP-1 production in some cells). ${ }^{28}$ In our present study, systolic blood pressure values correlated negatively with serum TSP-1 levels after one year of treatment (significant decreases in systolic blood pressure and heart rate after one year of treatment represent the most important cardiovascular protective factors). Positive correlation with decreases in systolic blood pressure further suggests that high levels of TSP-1 are necessary to maintain systemic vascular tone. $^{39}$

Although aging has been suggested to induce TSP-1 expression, ${ }^{40}$ multivariate analyses revealed that age greater than 56.5 years is not a risk factor for TSP-1 elevation. We endeavored to avoid possible circadian variations in TSP-1 levels as blood pressure monitoring and sampling were performed within 30 mins around $9 \mathrm{am}$.

In the activated endothelium, platelets form clots in association with components of the extracellular matrix, regulating localized angiogenesis. Two mechanisms are currently known for characterizing differential release of growth factors from platelets: the well-documented proteinase-activated system and the highly complex interactions of tissue-specific anchors such as heparan sulfate (less well documented). ${ }^{41,42}$ In the setting of a wound, the primary healing step is formation of an early vascular sprout is by VEGF activity. Basic fibroblast growth factor (bFGF) subsequently induces vessel growth and lumen development, followed by endothelial cell proliferation and structural stabilization with the aid of heparan sulfate binding proteins. The inhibition of excess angiogenesis is dependent

Table 7 Results Of The Logistic Regression Performed At Baseline (T0)

\begin{tabular}{|l|l|l|l|l|l|l|}
\hline TSP-I Risk/Protective Factor & \multirow{2}{*}{$\mathbf{P}^{\text {sign. }}$} & \multirow{2}{*}{ OR } & \multicolumn{2}{l|}{$\mathbf{9 5 \%}$ Interval Of Confidence } & \multicolumn{2}{l|}{ Spearman Correlation } \\
\cline { 4 - 7 } & & & Lower Limit & Upper Limit & $\rho$ & $\mathbf{P}^{\text {sign. }}$ \\
\hline BUN - protective factor & $0.005^{\mathrm{s}}$ & 0.009 & 0.01 & 0.7 & -0.204 & $0.015^{\mathrm{s}}$ \\
ALAT - risk factor & $0.004^{\mathrm{s}}$ & 11.34 & 1.42 & 90.42 & 0.416 & $<0.001^{\mathrm{s}}$ \\
Glucose - risk factor & $0.023^{\mathrm{s}}$ & 2.30 & 1.11 & 4.77 & 0.361 & $<0.001^{\mathrm{s}}$ \\
HR - risk factor & $0.011^{\mathrm{s}}$ & 3.3 & 1.27 & 8.57 & 0.170 & $0.044^{\mathrm{s}}$ \\
EDLV - risk factor & $0.046^{\mathrm{s}}$ & 2.77 & 1.72 & 7.76 & 0.081 & $0.337^{\mathrm{ns}}$ \\
RDW - risk factor & $0.016^{\mathrm{s}}$ & 3.32 & 1,2 & 9.13 & 0.159 & $0.059^{\mathrm{ns}}$ \\
MPV - risk factor & $0.026^{\mathrm{s}}$ & 4.06 & 1.19 & 15.09 & -0.094 & $0.267^{\mathrm{ns}}$ \\
DLA - risk factor & $0.013^{\mathrm{s}}$ & 4.71 & 1.25 & 17.71 & 0.238 & $0.004^{\mathrm{s}}$ \\
RBC - risk factor & $<0.001^{\mathrm{s}}$ & 8.16 & 2.27 & 29.33 & 0.345 & $<0.001^{\mathrm{s}}$ \\
MCH - protective factor & $0.015^{\mathrm{s}}$ & 0.11 & 0,01 & 0.9 & -0.234 & $0.005^{\mathrm{s}}$ \\
\hline
\end{tabular}

Abbreviations: BUN, blood urea nitrogen; ALAT, alanine aminotransferase (GTP); HR, heart rate; EDLV, end-diastolic diameter of left ventricle; RDW, Red cell distribution width; MPV, mean platelet volume; DLA, diameter of left atrium; RBC, red blood cells; MCH, mean corpuscular hemoglobin; S, significant; NS, non-significant. 
Table 8 Results Of Logistic Regression Performed After One Year Of Perindopril Treatment (TI)

\begin{tabular}{|c|c|c|c|c|c|c|}
\hline \multirow[t]{2}{*}{ TSP-I Risk/Protective Factor } & \multirow[t]{2}{*}{$\mathbf{P}^{\text {sign. }}$} & \multirow[t]{2}{*}{ OR } & \multicolumn{2}{|c|}{$\mathbf{9 5 \%}$ Interval Of Confidence } & \multicolumn{2}{|c|}{ Spearman Correlation } \\
\hline & & & Lower Limit & Upper Limit & $\rho$ & $\mathbf{p}^{\text {sign. }}$ \\
\hline BUN - protective factor & $0.002^{\mathrm{s}}$ & 0.20 & 0.07 & 0.56 & -0.422 & $<0.001^{s}$ \\
\hline ALAT- protective factor & $<0.001^{\mathrm{s}}$ & 0.18 & 0.09 & 0.43 & -0.44 & $0.723^{\text {ns }}$ \\
\hline Glucose - risk factor & $0.001^{\mathrm{s}}$ & 4,56 & 1.76 & 11.78 & -0.046 & $0.714^{\mathrm{ns}}$ \\
\hline $\mathrm{HR}$ - risk factor & $0.01^{\mathrm{s}}$ & 9.52 & 1.22 & 74.12 & 0.225 & $0.069^{\text {ns }}$ \\
\hline Potassium - protective factor & $0.002^{\mathrm{s}}$ & 0.07 & 0.01 & 0.62 & -0.479 & $<0.001^{\mathrm{s}}$ \\
\hline $\mathrm{MCH}$ - protective factor & $<0.001^{\mathrm{s}}$ & 0.22 & 0.02 & 0.18 & -0.633 & $<0.001^{\mathrm{s}}$ \\
\hline
\end{tabular}

Abbreviations: BUN, blood urea nitrogen; ALAT, alanine aminotransferase (GTP); HR, heart rate; MCH, mean corpuscular hemoglobin; S, significant; NS, non-significant.

on the activity of TSP-1 and platelet factor-4 (PF-4); both exhibit high affinity for heparan sulfate. The inhibitors of angiogenesis continue to be released under physiological conditions based on cell-cell interactions. ${ }^{41}$

The integrin-associated protein CD47 inhibits phagocytosis of erythrocytes by macrophages in the splenic and hepatic reticuloendothelial system by binding signal-regulatory protein alpha (SIRP $\alpha$ ) on macrophages. Burger et al reported evidence of a previously unknown mechanism for aged erythrocyte clearance via CD47-SIRP interaction in an animal model. High TSP-1 levels are thus likely involved in erythrocyte clearance by binding to CD47. This phenomenon explains the marked decrease in red blood cells, hemoglobin (HGB), hematocrit (HCT), red cell distribution width (RDW)-coefficient of variation (CV), RDW-standard deviation (SD) as well as the positive correlation between TSP-1, mean corpuscular hemoglobin, and red cell distribution width after one year of treatment with Perindopril. ${ }^{43}$ Decreases in HCT and HGB have been well documented by prior studies as well. ${ }^{44}$

This study had several limitations, namely a relative small study of patient population, an absence of exact molecular mechanism elucidation, a short period of observation, and a lack of circulating plasma TSP-1, endothelin-1, interleukin, and asymmetric dimethylarginine level quantification.

\section{Conclusion}

Current literature contains contradictory and incomplete data regarding the roles of TSP-1 in the cardiovascular system. Here, we assessed the relationship between serum levels of TSP-1, hypertension, endothelial dysfunction, and other relevant parameters both at baseline and after one year of Perindopril treatment.

Further research investigating the effects of ARBs on plasma TSP-1 levels in the setting of similar pathology is needed in order to establish a more accurate overview of commonly used drug mechanisms involved in the modulation of the renin-angiotensin-aldosterone system and pleiotropic properties on TSP-1 levels.

\section{Acknowledgments}

This work was supported by the grant PII-C4-TC-2016/ 16441-09 offered by the "Victor Babes" University of Medicine and Pharmacy of Timisoara, Romania.

\section{Disclosure}

The authors report no conflicts of interest in this work.

\section{References}

1. Krishna SM, Golledge J. The role of thrombospondin-1 in cardiovascular health and pathology. Int $J$ Cardiol. 2013;168(2):692-706. doi:10.1016/j.ijcard.2013.04.139

2. Lawler J. The functions of thrombospondin-1 and-2. CurrOpin Cell Biol. 2000;12(5):634-640.

3. Phelan MW, Forman LW, Perrine SP, Faller DV. Hypoxia increases thrombospondin-1 transcript and protein in cultured endothelial cells. $J$ Lab Clin Med. 1998;132(6):519-529.

4. Isenberg JS, Wink DA, Roberts DD. Thrombospondin-1 antagonizes nitric oxide-stimulated vascular smooth muscle cell responses. Cardiovasc Res. 2006;71(4):785-793. doi:10.1016/j.cardiores.2006. 05.024

5. Rogers NM, Yao M, Novelli EM, Thomson AW, Roberts DD, Isenberg JS. Activated CD47 regulates multiple vascular and stress responses: implications for acute kidney injury and its management. Am J Physiol Renal Physiol. 2012;303(8):F1117-25. doi:10.1152/ajprenal.00359

6. Wikner NE, Dixit VM, Frazier WA, Clark RA. Human keratinocytes synthesize and secrete the extracellular matrix protein, thrombospondin. J Invest Dermatol. 1987;88(2):207-211. doi:10.1111/1523-1747. ep12525350

7. Dameron KM, Volpert OV, Tainsky MA, Bouck N. Control of angiogenesis in fibroblasts by $\mathrm{p} 53$ regulation of thrombospondin-1. Science. 1994;265(5178):1582-1584. doi:10.1126/science. 7521539

8. Li SS, Ivanoff A, Bergström SE, et al. T lymphocyte expression of thrombospondin-1 and adhesion to extracellular matrix components. Eur J Immunol. 2002;32(4):1069-1079. doi:10.1002/1521-4141 (200204)32:4<1069::AID-IMMU1069>3.0.CO;2-E

9. Lessey-Morillon EC, Roberts DD. Thrombospondin-1: an extracellular message delivered by macrophages that promotes aortic aneurysms. Circ Res. 2015;117(2):113-115. doi:10.1161/CIRCRESAHA.117.306815 
10. Smith RE, Reyes NJ, Khandelwal P, et al. Secondary allergic T cell responses are regulated by dendritic cell-derived thrombospondin-1 in the setting of allergic eye disease. J Leukoc Biol. 2016;100(2):371380. doi:10.1189/jlb.3A0815-357RR

11. Tooney PA, Sakai T, Sakai K, Aeschlimann D, Mosher DF. Restricted localization of thrombospondin-2 protein during mouse embryogenesis: a comparison to thrombospondin-1. Matrix Biol. 1998;17(2):131-143.

12. Chen H, Herndon ME, Lawler J. The cell biology of thrombospondin-1. Matrix Biol. 2000;19(7):597-614.

13. Lopez-Dee Z, Pidcock K, Gutierrez LS. Thrombospondin-1: multiple paths to inflammation. Mediators Inflamm. 2011;296069. doi:10.11 55/2011/296069

14. Moura R, Tjwa M, Vandervoort P, Cludts K, Hoylaerts MF. Thrombospondin-1 activates medial smooth muscle cells and triggers neointima formation upon mouse carotid artery ligation. Arterioscler Thromb Vasc Biol. 2007;27(10):2163-2169.

15. Raman P, Krukovets I, Marinic TE, Bornstein P, Stenina OI Glycosylation mediates up-regulation of a potent antiangiogenic and proatherogenic protein, thrombospondin-1, by glucose in vascular smooth muscle cells. J Biol Chem. 2007;282(8):5704-5714. doi:10.1074/jbc.M610965200

16. Isenberg JS, Ridnour LA, Perruccio EM, Espey MG, Wink DA, Roberts DD. Thrombospondin-1 inhibits endothelial cell responses to nitric oxide in a cGMP-dependent manner. Proc Natl Acad Sci USA. 2005;102(37):13141-13146. doi:10.1073/pnas.0502977102

17. Vila V, Martínez-Sales V, Almenar L, Lázaro IS, Villa P, Reganon E. Inflammation, endothelial dysfunction and angiogenesis markers in chronic heart failure patients. Int $J$ Cardiol. 2008;130(2):276-277. doi:10.1016/j.ijcard.2007.07.010

18. Kuebler WM. What mediates the effects of thrombospondin-1 in pulmonary hypertension? New evidence for a dual-pronged role of CD47. Cardiovasc Res. 2017;113(1):3-5. doi:10.1093/cvr/cvw232

19. Roberts DD, Kaur S, Isenberg JS. Regulation of cellular redox signaling by matricellular proteins in vascular biology, immunology, and cancer. Antioxid Redox Signal. 2017;27(12):874-911. doi:10.1089/ars.2017.7140

20. Kumar R, Mickael C, Kassa B, et al. TGF- $\beta$ activation by bone marrowderived thrombospondin-1 causes Schistosoma- and hypoxia-induced pulmonary hypertension. Nat Commun. 2017;8:15494. doi:10.1038/ ncomms 15494

21. Thijssen DH, Black MA, Pyke KE, et al. Assessment of flowmediated dilation in humans: a methodological and physiological guideline. Am J Physiol Heart Circ Physiol. 2011;300(1):H2-H12. doi:10.1152/ajpheart.00471.2010

22. Miyamoto M, Kotani K, Ishibashi S, Taniguchi N. The effect of antihypertensive drugs on endothelial function as assessed by flowmediated vasodilation in hypertensive patients. Int $J$ Vasc Med. 2012;453264. doi:10.1155/2012/453264

23. Chen JD, Liu M, Chen XH, Yang ZJ. Effect of Angiotensin receptor blockers on flow-mediated vasodilation: a meta-analysis of randomized controlled trials. Cardiology. 2015;131(2):69-79. doi:10.1159/ 000375259

24. Buda V, Andor M, Petrescu L, et al. Perindopril induces TSP-1 expression in hypertensive patients with endothelial dysfunction in chronic treatment. Int $J$ Mol Sci. 2017;18(2):348. doi:10.3390/ ijms 18020348

25. Ghimire K, Altmann HM, Straub AC, Isenberg JS. Nitric oxide: what's new to NO? Am J Physiol Cell Physiol. 2017;312(3):C254C262. doi:10.1152/ajpcell.00315.2016

26. Bauer PM, Bauer EM, Rogers NM, et al. Activated CD47 promotes pulmonary arterial hypertension through targeting caveolin-1. Cardiovasc Res. 2012;93(4):682-693. doi:10.1093/cvr/cvr356

27. Csányi G, Yao M, Rodríguez AI, et al. Thrombospondin-1 regulates blood flow via CD47 receptor-mediated activation of NADPH oxidase 1. Arterioscler Thromb Vasc Biol. 2012;32(12):2966-2973. doi:10.1161/ATVBAHA.112.300031
28. Yao M, Rogers NM, Csányi G, et al. Thrombospondin-1 activation of signal-regulatory protein- $\alpha$ stimulates reactive oxygen species production and promotes renal ischemia reperfusion injury. $J \mathrm{Am}$ Soc Nephrol. 2014;25(6):1171-1186. doi:10.1681/ASN.2013040433

29. Remková A, Kratochvílová H, Durina J. Impact of the therapy by renin-angiotensin system targeting antihypertensive agents perindopril versus telmisartan on prothrombotic state in essential hypertension. $J$ Hum Hypertens. 2008;22(5):338-345. doi:10.1038/sj.jhh.1002328

30. Barclay JL, Keshvari S, Whitehead JP, Inder WJ. Development of an enzyme-linked immunosorbent assay for thrombospondin-1 and comparison of human plasma and serum concentrations. Ann Clin Biochem. 2016;53(Pt 5):606-610. doi:10.1177/0004563216628891

31. Buda V, Andor M, Cristescu C, et al. The influence of perindopril on PTX3 plasma levels in hypertensive patients with endothelial dysfunction. Farmacia. 2016;64(3):382-389.

32. Buda V, Andor M, Cristescu C, et al. The effect of candesartan on pentraxin-3 plasma levels as marker of endothelial dysfunction in patients with essential arterial hypertension. Ir J Med Sci. 2017;186 (3):621-629. doi:10.1007/s11845-017-1580-5

33. Buda V, Andor M, Baibata DE, et al. Decreased sEng plasma levels in hypertensive patients with endothelial dysfunction under chronic treatment with Perindopril. Drug Des Devel Ther. 2019;13:19151925. doi:10.2147/DDDT.S186378

34. Lamy L, Foussat A, Brown EJ, Bornstein P, Ticchioni M, Bernard A. Interactions between $\mathrm{CD} 47$ and thrombospondin reduce inflammation. $J$ Immunol. 2007;178:5930-5939. doi:10.4049/jimmunol.178.9.5930

35. Meng Y, Zhang M, Zhao X, et al. Decreased serum thrombospondin1 and elevation of its autoantibody are associated with multiple exacerbated clinical manifestations in systemic lupus erythematosus. ClinRheumatol. 2018. doi:10.1007/s10067-018-4188-x

36. Bauer EM, Qin Y, Miller TW, et al. Thrombospondin-1 supports blood pressure by limiting eNOS activation and endothelial-dependent vasorelaxation. Cardiovasc Res. 2010;88(3):471-481. doi:10.1093/cvr/cvq218

37. Wang S, Skorczewski J, Feng X, Mei L, Murphy-Ullrich JE. Glucose up-regulates thrombospondin 1 gene transcription and transforming growth factor-beta activity through antagonism of cGMP-dependent protein kinase repression via upstream stimulatory factor 2. J Biol Chem. 2004;279:34311-34322. doi:10.1074/jbc.M401629200

38. Zhao Y, Pu D, Sun Y, et al. High glucose-induced defective thrombospondin-1 release from astrocytes via TLR9 activation contributes to the synaptic protein loss. Exp Cell Res. 2018;363(2):171-178. doi:10.1016/j.yexcr.2017.12.030

39. Xia Y, Dobaczewski M, Gonzalez-Quesada C, et al. Endogenous thrombospondin 1 protects the pressure-overloaded myocardium by modulating fibroblast phenotype and matrix metabolism. Hypertension. 2011;58:902911. doi:10.1161/HYPERTENSIONAHA.111.175323

40. Cini C, Yip C, Attard C, et al. Differences in the resting platelet proteome and platelet releasate between healthy children and adults. $J$ Proteomics. 2015;123:78-88. doi:10.1016/j.jprot.2015.04.003

41. Kareva I, Abou-Slaybi A, Dodd O, Dashevsky O, Klement GL. Normal wound healing and tumor angiogenesis as a game of competitive inhibition. PLoS One. 2016;11(12):e0166655. doi:10.1371/journal.pone. 0166655

42. Ma L, Perini R, McKnight W, et al. Proteinase-activated receptors 1 and 4 counter-regulate endostatin and VEGF release from human platelets. Proc Natl Acad Sci U S A. 2005;102(1):216-220. doi:10.1073/pnas.0406682102

43. Burger P, Hilarius-Stokman P, de Korte D, van Den Berg TK, van Bruggen R. CD47 functions as a molecular switch for erythrocyte phagocytosis. Blood J. 2012;119:5512-5521. doi:10.1182/blood2011-10-386805

44. Spencer CG, Felmeden DC, Blann AD, Lip GY. Effects of "newer" and "older" antihypertensive drugs on hemorrheological, platelet, and endothelial factors. A substudy of the Anglo-Scandinavian Cardiac Outcomes Trial. Am J Hypertens. 2007;20(6):699-704. doi:10.1016/j. amjhyper.2007.01.004 


\section{Publish your work in this journal}

Drug Design, Development and Therapy is an international, peerreviewed open-access journal that spans the spectrum of drug design and development through to clinical applications. Clinical outcomes, patient safety, and programs for the development and effective, safe, and sustained use of medicines are a feature of the journal, which has also

been accepted for indexing on PubMed Central. The manuscript management system is completely online and includes a very quick and fair peer-review system, which is all easy to use. Visit http://www. dovepress.com/testimonials.php to read real quotes from published authors. 